\title{
Investigation of silicon on insulator fabricated by two-step $\mathrm{O}^{+}$ implantation
}

\author{
WEI Xing ${ }^{1,2^{*}}$, XUE ZhongYing ${ }^{1}$, WU AiMin ${ }^{1}$, WANG Xiang ${ }^{2}$, LI XianYuan ${ }^{2}$, YE Fei ${ }^{2}$, \\ CHEN Jie $^{2}$, CHEN Meng ${ }^{2}$, ZHANG Bo ${ }^{1}$, LIN ChengLu ${ }^{1}$, ZHANG Miao ${ }^{1,2^{*}}$ \& WANG Xi ${ }^{1,2}$ \\ ${ }^{1}$ State Key Laboratory of Functional Material for Informatics, Shanghai Institute of Microsystem and Information Technology, Chinese \\ Academy of Sciences, Shanghai 200050, China; \\ ${ }^{2}$ Shanghai Simgui Technology Company Limited, Shanghai 201821, China
}

Received November 6, 2009; accepted February 5, 2010

In this paper, we investigated the dose window of forming a continuous buried oxide (BOX) layer by single implantation at the implantation energy of $200 \mathrm{keV}$. Then, an improved two-step implantation process with second implantation dose of $3 \times 10^{15} \mathrm{~cm}^{-2}$ was developed to fabricate high quality separation by implanted oxygen (SIMOX) silicon on insulator (SOI) wafers. Compared with traditional single implantation, the implantation dose is reduced by $18.2 \%$. In addition, the thickness and uniformity of the BOX layers were evaluated by spectroscopic ellipsometry. Defect-free top $\mathrm{Si}$ as well as atomic-scale sharp top Si/buried oxide interfaces were observed by transmission electron microscopy, indicating a high crystal quality and a perfect structure of the SOI fabricated by two step implantation. The top Si/BOX interface morphology of the SOI wafers fabricated by single or two-step implantation was also investigated by atomic force microscopy.

silicon on insulator, interface morphology, dose window, separation by implanted oxygen

Citation: Wei X, Xue Z Y, Wu A M, et al. Investigation of silicon on insulator fabricated by two-step O ${ }^{+}$implantation. Chinese Sci Bull, 2011, 56: 444-448, doi: $10.1007 / \mathrm{s} 11434-011-4382-6$

Silicon-on-insulator wafers offer a number of advantages over bulk silicon wafers, such as high speed operation, low power consumption, anti-radiation, simple process and greater packaging densities. Now, it has been widely used in low-power/low-voltage and high-speed ultra-large-scale integrated (ULSI) circuits [1], micro-electro-mechanical system (MEMS) [2-4], high-voltage power devices [5] and optical waveguides [6]. Separation by implanted oxygen (SIMOX) is one of the leading techniques for synthesizing SOI wafers because it provides an excellent thickness uniformity of both the top $\mathrm{Si}$ and buried oxide (BOX) layers. Traditionally, the most widely used high dose SIMOX wafer is produced by implantation of more than $1.8 \times 10^{18} \mathrm{~cm}^{-2}$ ${ }^{16} \mathrm{O}^{+}$at $550^{\circ} \mathrm{C}$, which results in a BOX layer thickness of about $400 \mathrm{~nm}$. However, the high dose implantation gives

*Corresponding authors (email: xwei@mail.sim.ac.cn, mzhang@mail.sim.ac.cn) rise not only to a high density of threading dislocations in the top Si layer, but also to a high production cost. In recent years, there has been a growing interest in low/medium-dose SIMOX (dose $<1 \times 10^{18} \mathrm{~cm}^{-2}$ ) [7], with a BOX layer thickness of 50-200 nm, because of potential technological and economic advantages over high-dose SIMOX. These involve drastically reduced production cost and threading dislocations, improved device performance and thermal conductivity. For instance, it is reported that a thin BOX layer formed by low dose oxygen implantation is effective on reducing the self heating effect in metal-oxidesemiconductor field effect transistors [8]. However, as the implantation dose is reduced, the BOX layer tends to have discontinuities including breaks and high density of silicon islands. It is reported that $\mathrm{Si}$ islands are responsible for increased electrical leakage current through the BOX [9], or in extreme cases, its dielectric breakdown [10]. Many efforts 
have been made over the past years to fabricate high quality low dose SIMOX wafers $[7,11,12]$, such as internal oxidation [13-15] and dose energy match implantation [16-18], but they mainly focused on the optimization of the annealing process or dose windows at variable implantation energy. Apart from that, a novel technique is presented to improve the continuity of the BOX layers of SIMOX by introducing an additional ion-induced defect implantation step at room temperature [19]. This technique does not involve any variation of the post implantation annealing, but rather modifies the implantation process itself. It is shown that the SIMOX with a continuous BOX layer can be achieved by the two-step implantation process; however, the defects including twins and pyramidal stacking faults were observed at the top $\mathrm{Si} / \mathrm{BOX}$ interfacial region [19].

In this paper, we investigated the dose window for forming a continuous BOX layer at the implantation energy of $200 \mathrm{keV}$. Then, an improved two-step implantation process was proposed to fabricate high quality SIMOX SOI wafers. In addition, the authors investigated the cross-sectional structure of the SOI wafers by transmission electron microscopy (TEM). Finally, comparisons of the top Si/BOX interface morphology of the SOI wafers fabricated by single or two-step implantation were also discussed.

\section{Experimental details}

Oxygen ions (16O+) with doses of $3.6 \times 10^{17}, 4.0 \times 10^{17}$ and $4.4 \times 10^{17} \mathrm{~cm}^{-2}$ were implanted into $150-\mathrm{mm}$ p-type (100) Czochralski Si wafers at $200 \mathrm{keV}$. The wafer temperature was kept at $550^{\circ} \mathrm{C}$ during the implantation. Subsequently, the second implantation was performed at room temperature with the dose of $3 \times 10^{15} \mathrm{~cm}^{-2}$ at $190 \mathrm{keV}$. All the as-implanted wafers were annealed at $1350^{\circ} \mathrm{C}$ in a mixed atmosphere (5\% oxygen and $95 \%$ argon) for $8 \mathrm{~h}$. The BOX thickness uniformity of the wafers was evaluated by spectroscopic ellipsometry (SE). The authors also investigated the cross-sectional structure of the SIMOX wafers by transmission electron microscopy (TEM). The top Si layers of the SIMOX wafers were etched by a 5\% tetramethyl-ammonium hydroxide (TMAH) solution heated to $55^{\circ} \mathrm{C}$ until the upper surface of the BOX layers was exposed, and then, the top $\mathrm{Si} / \mathrm{BOX}$ interface morphology was characterized by atomic force microscopy (AFM).

\section{Results and discussion}

In order to evaluate the thickness uniformity of the BOX layers, all the samples were measured by SE at 49 locations over the entire wafers. The thickness variations of the BOX layers are shown in Figure 1.

As shown in curve $\mathrm{A}$, the BOX thickness variations of the SIMOX SOI wafers fabricated by single implantation

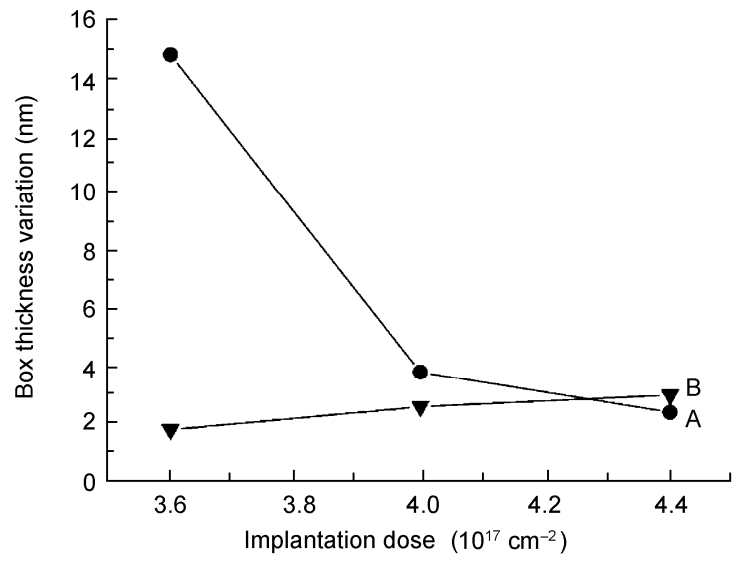

Figure 1 The relationships of BOX thickness variations versus implantation doses.

are demonstrated. Clearly, with the increase of the implantation dose, the thickness variations of the BOX layers are dramatically reduced from $14.8 \mathrm{~nm}$ to $2.4 \mathrm{~nm}$. These results indicate that a uniform BOX layer was formed at the implantation dose of $4.4 \times 10^{17} \mathrm{~cm}^{-2}$. It also can be confirmed by the result of cross-sectional electron transmission microscopy (XTEM).

The cross-sectional structure of the SOI wafer fabricated by single implantation with the dose of $4.4 \times 10^{17} \mathrm{~cm}^{-2}$ is shown in Figure 2(a). Clearly, the sandwich-like structure including the top $\mathrm{Si}$ layer, $\mathrm{BOX}$, and $\mathrm{Si}$ substrate can be easily distinguished, and the sharp interfaces can be readily observed. A high-resolution TEM image of the interfacial region between the top $\mathrm{Si}$ layer and the $\mathrm{BOX}$ is shown in Figure 2(b). An atomic-scale sharp interface without undulation is clearly demonstrated. No discernable defects can be observed, which indicates a high crystal quality of the top Si layer. It is well known that the dose window for forming a continuous BOX layer is about $4 \times 10^{17} \mathrm{~cm}^{-2}$ at $180 \mathrm{keV}$ [7]. In consideration of the strong dependence of implantation dose on acceleration energy [16-18], the implantation dose needed to form a continuous BOX layer at $200 \mathrm{keV}$ is more than $4 \times 10^{17} \mathrm{~cm}^{-2}$. Based on the results of SE and XTEM, it is suggested that the dose window at 200 $\mathrm{keV}$ is about $4.4 \times 10^{17} \mathrm{~cm}^{-2}$.

The single implantation dose of $3.6 \times 10^{17} \mathrm{~cm}^{-2}$ at 200 $\mathrm{keV}$ is far below the dose window of $4.4 \times 10^{17} \mathrm{~cm}^{-2}$. This implantation dose, therefore, is not sufficient to form a uniform BOX layer. The cross-sectional structure of the SOI wafer fabricated by single implantation with the dose of $3.6 \times 10^{17} \mathrm{~cm}^{-2}$ is shown in Figure 3 .

Local thinning of the BOX layer as well as interfacial undulation is clearly observed, verifying the BOX thickness variation of $14.8 \mathrm{~nm}$ measured by SE. However, the uniform BOX layers are achieved by two step implantation with the doses below the dose window. As shown in curve B of Figure 1, the BOX thickness variations of the SIMOX SOI wafers fabricated by two-step implantation are demonstrated. 
(a)

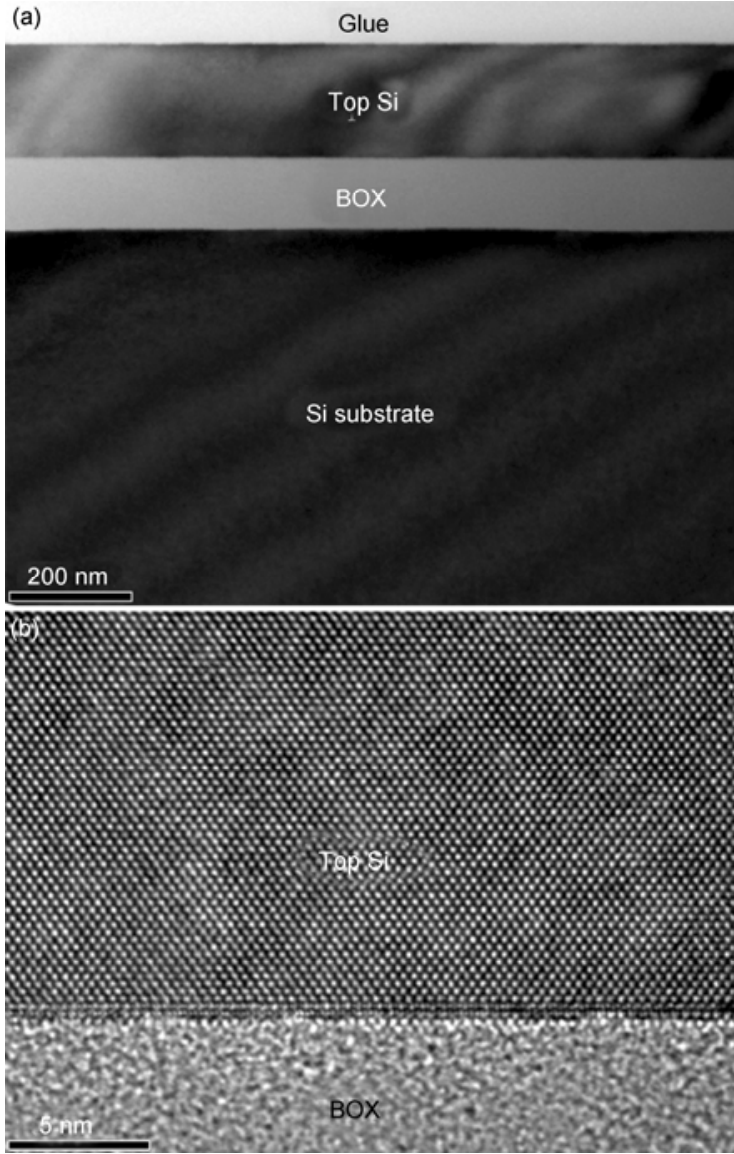

Figure 2 (a) XTEM image of the SOI fabricated by single implantation with the dose of $4.4 \times 10^{17} \mathrm{~cm}^{-2}$; (b) high-resolution TEM image of the top $\mathrm{Si} / \mathrm{BOX}$ interfacial region.

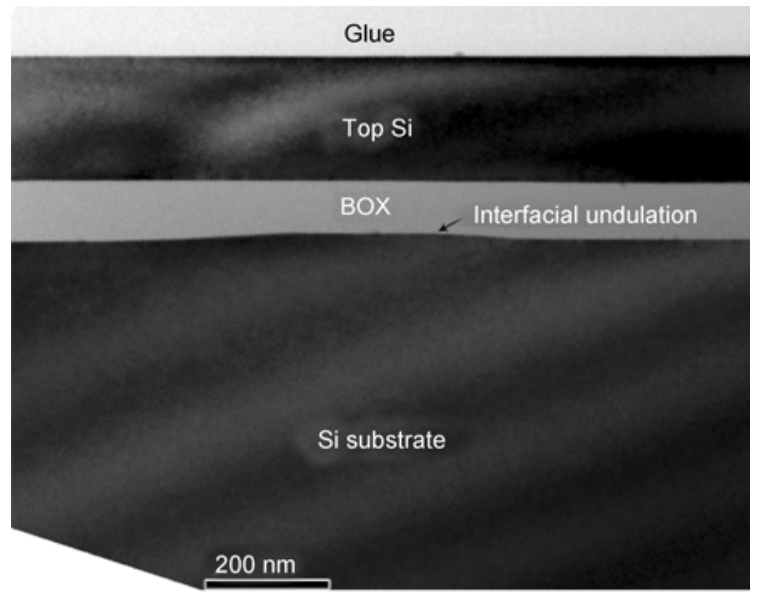

Figure 3 XTEM image of the SOI fabricated by single implantation with the dose of $3.6 \times 10^{17} \mathrm{~cm}^{-2}$. Note that interfacial undulation can be observed.

With the increase of the implantation dose, the thickness variations of the BOX layers are slightly increased from 1.8 to $3.0 \mathrm{~nm}$. All these values are comparable to that of the SOI wafer fabricated by single implantation with the dose of $4.4 \times 10^{17} \mathrm{~cm}^{-2}$, indicating uniform BOX layers are achieved by two step $\mathrm{O}^{+}$implantation.

The cross-sectional structure of the SOI wafer fabricated by two step implantation with the dose of $3.63 \times 10^{17} \mathrm{~cm}^{-2}$ is shown in Figure 4(a). A uniform BOX layer and sharp interfaces are observed, revealing the perfect structure of the SOI wafer. Compared with the dose window of $4.4 \times 10^{17}$ $\mathrm{cm}^{-2}$, the implantation dose is reduced by $18.2 \%$, indicating effective decrease of implantation dose achieved by the two-step implantation. Compared with the SOI fabricated by single implantation with the dose of $3.6 \times 10^{17} \mathrm{~cm}^{-2}$, the BOX thickness of the SOI fabricated by two step implantation with the dose of $3.63 \times 10^{17} \mathrm{~cm}^{-2}$ increases from 91.1 to $123.8 \mathrm{~nm}$, while the thickness variation decreases from 14.8 to $1.8 \mathrm{~nm}$, respectively. The difference between these two samples in thickness and uniformity of the BOX layers can be explained by the different implantation processes. It is reported that the ion-induced defect morphology near the ions' range $\left(R_{\mathrm{p}}\right)$ caused by second implantation is effective to facilitate or promote the formation of a high quality BOX layer [19]. During the second implantation at room temperature, the region near $R_{\mathrm{p}}$ (where the $\mathrm{BOX}$ forms during subsequent annealing) is selectively amorphized so that the affected layer will recrystallize during the post-impla-

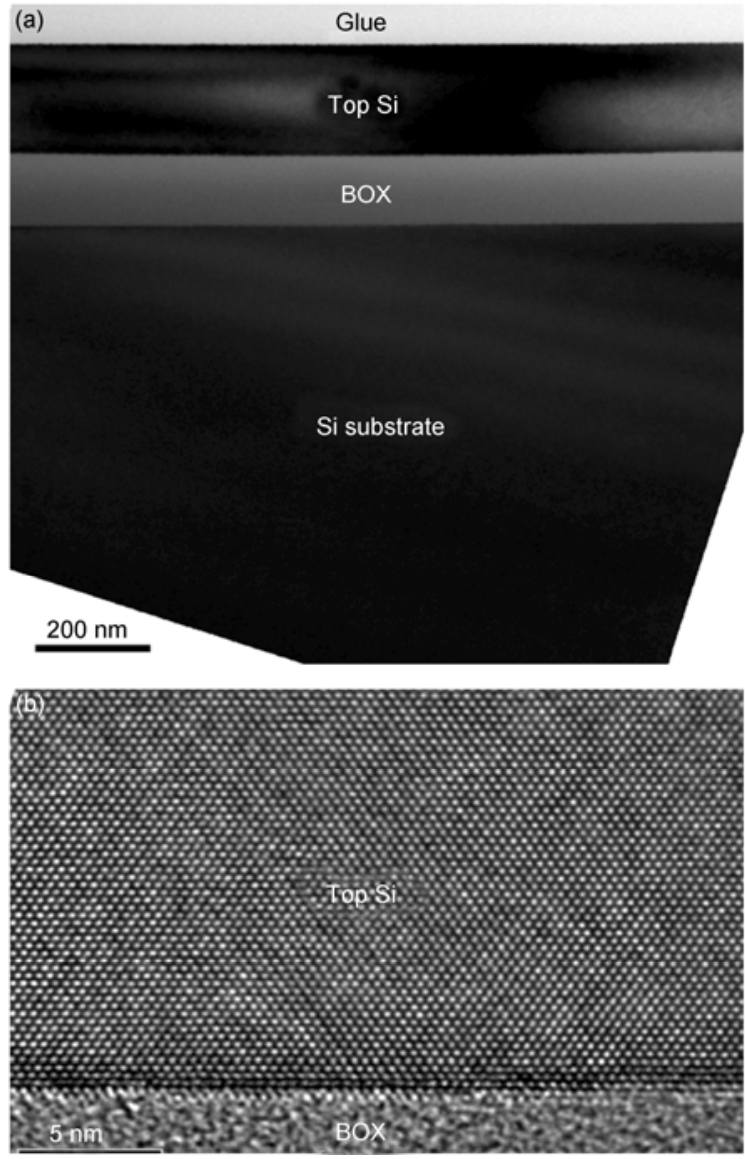

Figure 4 (a) XTEM image of the SOI fabricated by two-step implantation with the dose of $3.63 \times 10^{17} \mathrm{~cm}^{-2}$; (b) high-resolution TEM image of the top $\mathrm{Si} / \mathrm{BOX}$ interfacial region. 
ntation annealing to form polycrystal. Diffusion of oxygen is promoted along the grain boundaries of the polycrystal within the layer, and it is thought to facilitate the formation of the BOX layer during high temperature annealing [19].

Moreover, the process optimization of the two-step implantation was also achieved in this experiment. In Holland's work, the second implantation with the dose of $1 \times 10^{15} \mathrm{~cm}^{-2}$ was found to be optimum for forming a polycrystalline layer spatially matched to the desired BOX. Defects including twins and pyramidal stacking faults were still observed at the top $\mathrm{Si} / \mathrm{BOX}$ interfacial region of the SOI wafer [19]. However, the second implantation with the dose of $3 \times 10^{15} \mathrm{~cm}^{-2}$ was performed in this experiment. As shown in Figure 4(a), sharp and straight interfaces can be observed. Also in Figure 4(b), defect-free top Si as well as atomic-scale sharp top $\mathrm{Si} / \mathrm{BOX}$ interface without undulation was demonstrated, manifesting a higher crystal quality of the top Si than that of Holland's work.

The interface quality of the top $\mathrm{Si} / \mathrm{BOX}$ layers significantly influences the performance of devices especially for fully depleted CMOS devices built on SOI wafers. For instance, the rough interface scatters the electrons and further results in electron mobility and lifetime degradation.

The upper BOX surface morphology of the SOI fabricated by single implantation with the dose of $4.4 \times 10^{17} \mathrm{~cm}^{-2}$ is shown in Figure 5, while that of the SOI fabricated by two-step implantation with the dose of $3.63 \times 10^{17} \mathrm{~cm}^{-2}$ is shown in Figure 6. The corresponding root-mean-square (RMS) roughness (calculated from a $5 \mu \mathrm{m} \times 5 \mu \mathrm{m}$ scan area) is $0.659 \mathrm{~nm}$ and $1.266 \mathrm{~nm}$, respectively. Clearly, the RMS roughness of upper BOX surface of the SOI fabricated by two-step implantation is slightly higher than that of the SOI fabricated by single implantation. But it is still much better than that of high dose SIMOX wafers (usually more than $2.5 \mathrm{~nm}$ ). Additionally, compared with Figure 5, more square

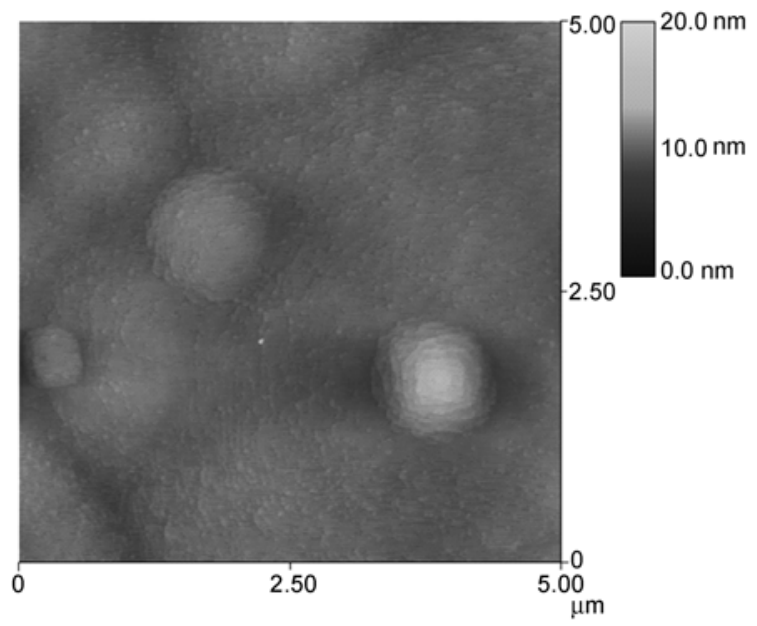

Figure 5 AFM image of the upper BOX surface of the SOI fabricated by single implantation with the dose of $4.4 \times 10^{17} \mathrm{~cm}^{-2}$.

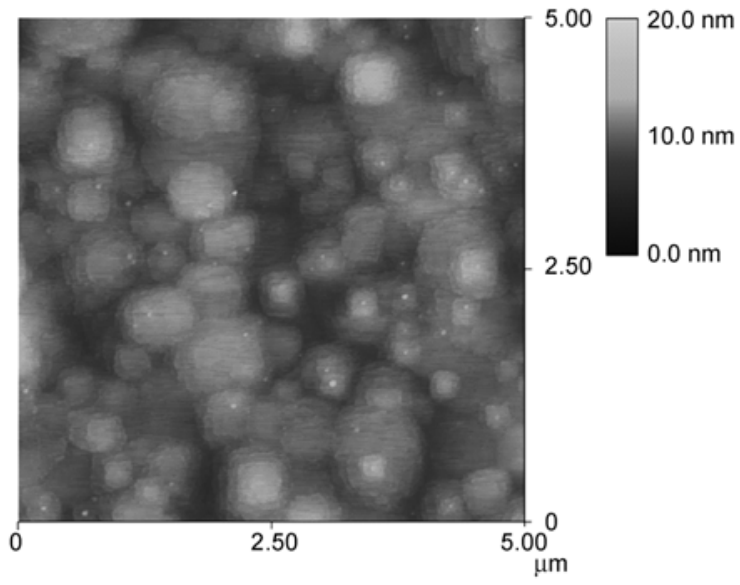

Figure 6 AFM image of the upper BOX surface of the SOI fabricated by two-step implantation with the dose of $3.63 \times 10^{17} \mathrm{~cm}^{-2}$.

mosaics are observed in Figure 6. This morphology is belived to reflect the grains of the polycrystal. The $\mathrm{Si}$ near $R_{\mathrm{p}}$ is selectively amorphized due to the second implantation. In the process of the following annealing, the polycrystal is formed by the recrystallization of the amorphized Si. Meanwhile, the square mosaics are formed by complete consumption of the grains of the polycrystal by orientation-dependent oxidation. The square mosaics morphology can be greatly improved by optimizing the annealing process of SIMOX, because the convex square mosaics can be eliminated after a sufficient period of annealing [20].

\section{Conclusions}

In this paper, $4.4 \times 10^{17} \mathrm{~cm}^{-2}$ at $200 \mathrm{keV}$ was found to be the dose window for forming a uniform BOX layer, and the BOX thickness variation of the obtained SIMOX wafer is $\pm 1.2 \mathrm{~nm}$. The improved two-step implantation process was presented to fabricate high quality SIMOX wafer, and the first implantation with dose of $3.6 \times 10^{17} \mathrm{~cm}^{-2}$ and the second implantation with dose of $3.0 \times 10^{15} \mathrm{~cm}^{-2}$ were performed at $200 \mathrm{keV}$ and $190 \mathrm{keV}$, respectively. SE results manifest that the SIMOX wafer with the BOX layer of $123.8 \mathrm{~nm} \pm 0.9 \mathrm{~nm}$ is achieved successfully by two-step implantation process. The results of TEM show sharp interfaces and defect-free top Si layer, revealing the perfect structure of the SOI wafer fabricated by two-step implantation process. AFM result shows a much better top $\mathrm{Si} / \mathrm{BOX}$ interface morphology than that of high dose SIMOX wafers. More importantly, compared with the dose window of the traditional single implantation, the implantation dose is reduced by $18.2 \%$, indicating effective reduction of implantation dose achieved by the two-step implantation. Therefore, two-step implantation process is a more competitive approach to fabricate high-quality SIMOX SOI wafers. 
This work was supported by the National Science and Technology Major Projects of China (2009ZX02040), the National Basic Research Program of China (2010CB832906), the National Natural Science Foundation of China (60721004 and 61006088), Shanghai Foundation for Development of Science and Technology (08520740100) and the Natural Science Foundation of Shanghai (10ZR1436100).

1 Pelloie J L, Auberton-Herve A. A new generation of IC processing: Low-power, high-performance SOI CMOS. Solid State Technol, 2001, 44: 63

2 Wu A M, Chen J, Wang X. A very sensitive pressure sensor on a SOI-on-cavity substrate. In: Proceedings of the 2007 IEEE International SOI Conference, 2007, 151-152

3 Pei W H, Zhu L, Wang S J, et al. Multi-channel micro neural probe fabricated with SOI. Sci China Ser E-Tech Sci, 2009, 52: 1187-1190

4 Jin Q H, Wang Y L, Li T, et al. A MEMS device for in-situ TEM test of SCS nanobeam. Sci China Ser E-Tech Sci, 2008, 51: 1491-1496

5 Zingg R P. High-voltage, double-gate devices on silicon-on-insulator. Microelectro Eng, 2001, 59: 461-468

6 Wang Y J, Lin Z L, Cheng X L, et al. Scattering loss in silicon-on-insulator rib waveguides fabricated by inductively coupled plasma reactive ion etching. Appl Phys Lett, 2004, 85: 3995-3997

7 Nakashima S, Izumi K. Analysis of buried oxide layer formation and mechanism of threading dislocation generation in the substoichiometric oxygen dose region. J Mater Res, 1993, 8: 523-534

8 Ohno T, Kado Y, Harada M, et al. Experimental $0.25-\mu \mathrm{m}$-gate fully depleted CMOS/SIMOX process using a new two-step LOCOS isolation technique. IEEE Trans Electron Dev, 1995, 42: 1481-1486

9 Yap J H, Nee J, Simic E, et al. A model for high-field conduction in SIMOX buried oxides. In: Proceedings of the 1994 IEEE International SOI Conference, 1994, 93

10 Nakashima S, Harada M, Tsuchiya T. Improvement of the breakdown field of SIMOX buried oxide layers. In: Proceedings of the 1993 IEEE. International SOI Conference, 1993, 14-15

11 Hamaguchi I, Fujita T, Yano T S, et al. Dislocation density reduction in SIMOX (Separation by Implanted Oxygen) multi-energy single implantation. Jpn J Appl Phys Part 1, 1995, 34: 2989-2993

12 Chen M, Chen J, Zheng W, et al. Effect of implantation energy on the microstructure evolution of low dose separation of implanted oxygen wafers. J Vac Sci Technol B, 2001, 19: 337-343

13 Ericsson P, Bengtsson S. Internal oxidation of low dose separation by implanted oxygen wafers in different oxygen/nitrogen mixtures. Appl Phys Lett, 1997, 71: 2310-2312

14 Schroer E, Hopfe S, Tong Q Y, et al. Growth of buried oxide layers of silicon-on-insulator structures by thermal oxidation of the top silicon layer. J Electrochem Soc, 1997, 144: 2205-2210

15 Nakashima S, Katayama T, Miyamura $\mathrm{Y}$, et al. Investigations on high-temperature thermal oxidation process at top and bottom interfaces of top silicon of SIMOX wafers. J Electrochem Soc, 1996, 143: 244-251

16 Chen M, Wang X, Chen J, et al. Dose-energy match for the formation of high-integrity buried oxide layers in low-dose separation-by-implantation-of-oxygen materials. Appl Phys Lett, 2002, 80: 880-882

17 Chen M, Wang X, Chen J, et al. Fabrication of device-grade silicon-on-insulator material from appropriate matches of low oxygen implantation dose and acceleration energy. J Vac Sci Technol B, 2003, 21: 2001-2010

18 Chen M, Yu Y H, Wang X, et al. Fabrication of device-grade separation-by-implantation-of-oxygen materialsby optimizing dose-energy match. J Mater Res, 2002, 17: 1634-1643

19 Holland O W, Fathy D, Sadana D K. Formation of ultrathin, buried oxides in Si by O ${ }^{+}$ion implantation. Appl Phys Lett, 1996, 69: 674-676

20 Ishiyama T, Omura Y. Analysis of interface microstructure evolution in separation by implanted oxygen (SIMOX) wafers. Jpn J Appl Phys Part 1, 2000, 39: 4653-4656

Open Access This article is distributed under the terms of the Creative Commons Attribution License which permits any use, distribution, and reproduction in any medium, provided the original author(s) and source are credited 